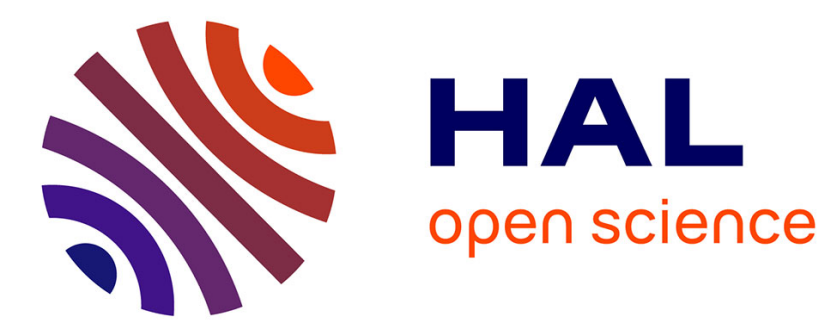

\title{
Volume functions on blow-ups and Seshadri constants
} François Ballaÿ

\section{To cite this version:}

François Ballaÿ. Volume functions on blow-ups and Seshadri constants. Proceedings of the American Mathematical Society, 2022, 150 (5), pp.1925-1935. hal-03322210

\section{HAL Id: hal-03322210 https://hal.science/hal-03322210}

Submitted on 18 Aug 2021

HAL is a multi-disciplinary open access archive for the deposit and dissemination of scientific research documents, whether they are published or not. The documents may come from teaching and research institutions in France or abroad, or from public or private research centers.
L'archive ouverte pluridisciplinaire HAL, est destinée au dépôt et à la diffusion de documents scientifiques de niveau recherche, publiés ou non, émanant des établissements d'enseignement et de recherche français ou étrangers, des laboratoires publics ou privés. 


\title{
VOLUME FUNCTIONS ON BLOW-UPS AND SESHADRI CONSTANTS
}

\author{
FRANÇOIS BALLAŸ \\ Université Clermont Auvergne, CNRS, LMBP, F-63000 Clermont-Ferrand, France. \\ francois.ballay@uca.fr
}

\begin{abstract}
We exhibit some relations between the Seshadri constant of an ample divisor along a closed subscheme and the behaviour of the volume function on the corresponding blow-up. As an application, we give an equivalent formulation of Nagata's conjecture in terms of the differentiability of a real valued function.
\end{abstract}

2020 Mathematics Subject Classification: 14C20.

Keywords: Seshadri constants, volume of divisors, Nagata's conjecture.

\section{INTRODUCTION}

Let $X$ be a projective variety of dimension $d \geq 1$ over an algebraically closed field $k$. The volume of a Cartier divisor $D$ on $X$ measures the asymptotic growth of the linear systems $|n D|, n \in \mathbb{N}$ :

$$
\operatorname{vol}(D)=\limsup _{n \rightarrow \infty} \frac{h^{0}(X, n D)}{n^{d} / d !} .
$$

This invariant depends only on the numerical equivalence class of $D$, and can be extended to define a continuous function

$$
\text { vol: } N^{1}(X)_{\mathbb{R}} \rightarrow \mathbb{R}
$$

on the real Néron-Severi group $N^{1}(X)_{\mathbb{R}}=N^{1}(X) \otimes_{\mathbb{Z}} \mathbb{R}$ of $X$ (see [12, section 2.2.C] and [5, section 2.4] for details). The big cone $\operatorname{Big}(X) \subseteq N^{1}(X)_{\mathbb{R}}$ is the convex cone of classes $\alpha \in N^{1}(X)_{\mathbb{R}}$ with $\operatorname{vol}(\alpha)>0$. The study of the volume function is an interesting topic of research, as emphasized in the survey [9]. For example, it is believed that vol is real analytic on some "large" open subset of the big cone [9, Conjecture 2.18]. The differentiability of vol on $\operatorname{Big}(X)$ was established by Boucksom, Favre and Jonsson [3] in characteristic zero and by Cutkosky [5] in general.

In this paper we are interested in the behaviour of the volume function on blowups. More precisely, let $D$ be an ample $\mathbb{R}$-Cartier $\mathbb{R}$-divisor on $X$ and let $Z \mp X$ be a closed proper subscheme, with defining ideal $I_{Z}$. Let $\pi_{Z}: \widetilde{X} \rightarrow X$ be the blow up along $I_{Z}$ and let $E=\pi_{Z}^{-1}(Z)$ be the exceptional divisor, with the scheme structure defined by the ideal $I_{Z} \mathcal{O}_{\widetilde{X}} \simeq \mathcal{O}_{\widetilde{X}}(1)$. For $t \in \mathbb{R}$, we let $D_{t}=\pi_{Z}^{*} D-t E$. The purpose of this short article is to observe that the behaviour of the function

$$
\varphi_{D, Z}: \mathbb{R}_{\geq 0} \rightarrow \mathbb{R}, t \mapsto \operatorname{vol}\left(D_{t}\right)
$$

is closely related to the Seshadri constant of $D$ along $Z$, defined to be

$$
\epsilon_{Z}(D)=\sup \left\{t \in \mathbb{R} \mid D_{t} \text { is ample }\right\} .
$$


Seshadri constants were originally introduced by Demailly [7] in the case where $Z=\{x\}$ is a closed point. They have been widely studied since then, at least when $\operatorname{dim} Z=0$, and have become an important tool to understand the geometry of projective varieties. We refer the reader to [2] and [12, Chapter 5] for background and motivation about this invariant. We shall prove the following theorem and study some of its consequences.

Theorem 1.1. Assume that the line bundle $\mathcal{O}_{E}(-E)=\mathcal{O}_{\widetilde{X}}(1)_{\mid E}$ is nef on $E$. Then

$$
\epsilon_{Z}(D)=\sup \left\{t \geq 0 \mid \varphi_{D, Z} \text { is polynomial on }[0, t]\right\} \text {. }
$$

We remark that the assumption on $\mathcal{O}_{E}(-E)$ is automatically satisfied when $Z=\left\{x_{1}, \ldots, x_{r}\right\}$ is a collection of $r \geq 1$ distinct closed points in $X$, since in that case the line bundle $\mathcal{O}_{\widetilde{X}}(1)$ is relatively ample. When the inclusion $Z \varsubsetneqq X$ is a regular embedding (e.g. when $X$ and $Z$ are smooth), $\mathcal{O}_{E}(-E)$ is nef if and only if the anti-conormal bundle $I_{Z} / I_{Z}^{2}$ is nef. We shall actually prove a generalization of Theorem 1.1, valid without any positivity assumption on $\mathcal{O}_{E}(-E)$ (see Theorem 3.1). The proof of Theorem 1.1 builds on ideas of McKinnon and Roth [13], and is based on a criterion for amplitude in terms of higher cohomological functions due to de Fernex, Küronya and Lazarsfeld [6] and to Murayama [15] (see Theorem 2.1 below). We use the assumption on $\mathcal{O}_{E}(-E)$ to guarantee the vanishing of the higher cohomological functions $\widehat{h}^{i}\left(\widetilde{X}, D_{t}\right)$ when $i \geq 2$ and $t \geq 0$ (see Lemma 3.3), in order to combine the main result of [6] with the asymptotic Riemann-Roch theorem. This leads to the identities

$$
\epsilon_{Z}(D)=\sup \left\{t \geq 0 \mid \operatorname{vol}\left(D_{t}\right)=D_{t}^{\operatorname{dim} X}\right\}=\sup \left\{t \geq 0 \mid \widehat{h}^{1}\left(\tilde{X}, D_{t}\right)=0\right\},
$$

from which we easily deduce Theorem 1.1.

Let $\gamma_{Z}(D)$ be the supremum of the real numbers $t$ such that $\varphi_{D, Z}(t)>0$. We consider the function

$$
\phi_{D, Z}:\left[0, \gamma_{Z}(D)\right] \rightarrow \mathbb{R}, t \mapsto\left\langle D_{t}^{\operatorname{dim} X-1}\right\rangle \cdot E,
$$

where $\left\langle D_{t}^{\operatorname{dim} X-1}\right\rangle$ denotes the positive intersection product introduced in $[3$, section 2] (see also [5, section 4]). By [5, Theorem 5.6], the function $\varphi_{D, Z}$ is differentiable on $\left(0, \gamma_{Z}(D)\right)$ and its derivative is given by $\varphi_{D, Z}^{\prime}=-\operatorname{dim}(X) \phi_{D, Z}$. In particular, Theorem 1.1 remains true if $\varphi_{D, Z}$ is replaced by $\phi_{D, Z}$.

Corollary 1.2. If $\mathcal{O}_{E}(-E)$ is nef, then

$$
\epsilon_{Z}(D)=\sup \left\{t \geq 0 \mid \phi_{D, Z} \text { is polynomial on }[0, t]\right\} \text {. }
$$

When $\tilde{X}$ is a smooth surface (for example when $X$ and $Z$ are smooth and $\operatorname{dim} X=2), \varphi_{D, Z}$ is a piecewise polynomial function of degree at most 2 by a theorem of Bauer, Küronya and Szemberg [1, Theorem]. It follows that $\varphi_{D, Z}$ and $\phi_{D, Z}$ are polynomial on an interval $[0, t]$ if and only if $\phi_{D, Z}$ is differentiable on $(0, t)$.

Corollary 1.3. Assume that $\widetilde{X}$ is a smooth surface and that $\mathcal{O}_{E}(-E)$ is nef. Then

$$
\epsilon_{Z}(D)=\sup \left\{t \geq 0 \mid \phi_{D, Z} \text { is differentiable on }(0, t)\right\} \text {. }
$$

It is worth to note that when $\widetilde{X}$ is a smooth surface, the function $\phi_{D, Z}$ is simply given by $\phi_{D, Z}(t)=P_{t} \cdot E$ for all $t \in\left[0, \gamma_{Z}(D)\right)$, where $P_{t}$ is the positive part in the Zariski decomposition of $D_{t}=\pi_{Z}^{*} D-t E$. This follows from the definition of the positive intersection product, as explained in [3, section 3.4]. Let us also mention that when $X$ is a smooth surface and $Z \varsubsetneqq X$ is a smooth irreducible curve, Theorem 1.1 and Corollary 1.3 remain valid even if $\mathcal{O}_{E}(-E)$ is not nef (see Corollary 3.5).

Let us now focus on zero-dimensional subschemes, that is $Z=\left\{x_{1}, \ldots, x_{r}\right\}$ is a collection of closed points in $X$. When $k$ is uncountable and the points $x_{1}, \ldots, x_{r}$ 
are in very general position, we shall see that the function $\varphi_{D, Z}$ depends only on $D$ and $r$ (see Proposition 4.2 for a precise statement, and Proposition 4.1 for a more general result valid for higher dimensional subschemes). We denote it by $\varphi_{D, r}$. It is differentiable on some non-empty interval $\left[0, \gamma_{r}(D)\right)$, and we let $\phi_{D, r}=$ $-\varphi_{D, r}^{\prime} / \operatorname{dim} X$. In particular, we recover from Theorem 1.1 the well-known fact that Seshadri constants at $r$ very general points all take the same value $\epsilon_{r}(D)$. Indeed, we have

$$
\epsilon_{r}(D)=\sup \left\{t \geq 0 \mid \varphi_{D, r} \text { is polynomial on }[0, t]\right\} .
$$

Of course we also have analogues of Corollaries 1.2 and 1.3: for example, if $X$ is a smooth surface then

$$
\epsilon_{r}(D)=\sup \left\{t \geq 0 \mid \phi_{D, r} \text { is differentiable on }(0, t)\right\} .
$$

A celebrated conjecture of Nagata predicts that for $r \geq 9$ very general points $x_{1}, \ldots, x_{r}$ in $\mathbb{P}_{\mathbb{C}}^{2}$ and for any integral curve $C \subset \mathbb{P}_{\mathbb{C}}^{2}$, we have

$$
\operatorname{deg}(C) \geq \frac{1}{\sqrt{r}} \sum_{i=1}^{r} \operatorname{mult}_{x_{i}} C .
$$

This conjecture was settled by Nagata when $r$ is a perfect square, but the general case remains open despite many attempts in the past decades. It can be formulated in terms of Seshadri constants as follows: if $D$ is a line in $\mathbb{P}_{\mathbb{C}}^{2}$, then $\epsilon_{r}(D) \geq 1 / \sqrt{r}$ whenever $r \geq 9$ ([12, Remark 5.1.14]). In view of (1), we have the following equivalent formulation of

Nagata's conjecture. Let $X=\mathbb{P}_{\mathbb{C}}^{2}$ and $D$ be a line. Then for any $r \geq 9$, the function $\phi_{D, r}$ is differentiable on $(0,1 / \sqrt{r})$.

We mention that similar reformulations of Nagata's conjecture can also be derived from the computation of Newton-Okounkov bodies of line bundles on blow-ups of $\mathbb{P}_{\mathbb{C}}^{2}$ obtained by Eckl ([8, Theorems 3.4 and 3.5]).

Organization of the paper. We recall some preliminary results on higher cohomological functions in section 2. We then prove Theorem 1.1 in section 3 . In section 4 we study the behaviour of $\varphi_{D, Z}$ when $Z$ varies in a flat family of subschemes (Proposition 4.1). In the case $\operatorname{dim} Z=0$, we prove that the function $\varphi_{D, Z}=\varphi_{D, r}$ depends only on $D$ and $r=\operatorname{card}(Z)$ when $Z$ consists of very general points (Proposition $4.2)$.

\section{Conventions And BACKGround}

Throughout this paper we work over an algebraically closed field $k$. We denote by $\operatorname{Div}(X)$ the group of Cartier divisors on a projective scheme $X$. A $\mathbb{R}$-Cartier $\mathbb{R}$-divisor on $X$ is an element of $\operatorname{Div}(X)_{\mathbb{R}}:=\operatorname{Div}(X) \otimes_{\mathbb{Z}} \mathbb{R}$. We let $N^{1}(X)_{\mathbb{R}}$ be the real Néron-Severi space of $X([12$, section $1.3 . \mathrm{B}])$. A projective variety is a reduced and irreducible projective scheme.

2.1. Higher cohomological functions. Let $X$ be a projective variety of dimension $d \geq 1$. For any integer $0 \leq i \leq d$, we denote by $\widehat{h}^{i}: \operatorname{Div}(X) \rightarrow \mathbb{R}$ the higher cohomological function introduced by Küronya in [11]: for any $D \in \operatorname{Div}(X)$ we have

$$
\widehat{h}^{i}(X, D)=\limsup _{n \rightarrow+\infty} \frac{h^{i}(X, n D)}{n^{d} / d !},
$$

where $h^{i}(X, n D)=h^{i}\left(X, \mathcal{O}_{X}(n D)\right)$ is the dimension of $H^{i}\left(X, \mathcal{O}_{X}(n D)\right)$ as a $k$ vector space. Note that $\widehat{h}^{0}(X, D)=\operatorname{vol}(D)$ coincides with the volume of $D$. Moreover, the functions $\widehat{h}^{i}$ are homogeneous of degree $d$ and induce well defined functions

$$
\widehat{h}^{i}: \operatorname{Div}(X)_{\mathbb{R}} \rightarrow \mathbb{R}
$$


for $0 \leq i \leq d$, that are continuous on every finite-dimensional $\mathbb{R}$-linear subspace with respect to any norm (see [11, Corollary 5.3] and [4, Proposition 3.4.8]). We will use the following theorem characterizing ampleness in terms of vanishing of higher cohomological functions. It was proved by de Fernex, Küronya and Lazarsfeld [6, Theorem A] over the complex numbers, and generalized in arbitrary characteristic by Murayama [15].

Theorem 2.1 ([15], Theorem B). Let $D, A \in \operatorname{Div}(X)_{\mathbb{R}}$ be two $\mathbb{R}$-Cartier $\mathbb{R}$-divisors on $X$, with $A$ ample. Then $D$ is ample if and only if there exists a real $\gamma>0$ such that

$$
\widehat{h}^{i}(X, D-t A)=0
$$

for all $i \in\{1, \ldots, d\}$ and $t \in(0, \gamma)$.

2.2. Cohomology of nef divisors. We need the following technical lemma for the proof of Theorem 1.1.

Lemma 2.2. Let $A_{1}, \ldots, A_{\ell} \in \operatorname{Div}(X)$ be nef Cartier divisors on a projective scheme $X$ of dimension $d \geq 0$. Then for any coherent sheaf $\mathcal{F}$ on $X$, there exists a real number $C$ such that

$$
h^{i}\left(X, \mathcal{O}_{X}\left(n_{1} A_{1}+\ldots+n_{\ell} A_{\ell}\right) \otimes_{\mathcal{O}_{X}} \mathcal{F}\right) \leq C \max _{1 \leq j \leq \ell} n_{j}^{d-i}
$$

for any integers $i, n_{1}, \ldots, n_{\ell} \geq 0$.

Proof. We adapt the arguments of [12, Theorem 1.4.40]. We may assume by induction that the statement is true for any projective scheme of dimension $\leq d-1$. For any $\mathbf{n}=\left(n_{1}, \ldots, n_{\ell}\right) \in \mathbb{N}^{\ell}$, we let $\mathbf{n} A \in \operatorname{Div}(X)$ be the nef Cartier divisor given by $\mathbf{n} A=\sum_{j=1}^{\ell} n_{j} A_{j}$. By Fujita's vanishing theorem [12, Theorem 1.4.35 and Remark 1.4.36], there exists a very ample divisor $H \in \operatorname{Div}(X)$ which does not contain any subvariety of $X$ defined by the associated primes of $\mathcal{F}$ and such that

$$
H^{i}\left(X, \mathcal{O}_{X}(H+\mathbf{n} A) \otimes_{\mathcal{O}_{X}} \mathcal{F}\right)=0
$$

for any $i \geq 1$ and $\mathbf{n} \in \mathbb{N}^{\ell}$. We have an exact sequence of sheaves

$$
0 \rightarrow \mathcal{O}_{X}(\mathbf{n} A) \otimes_{\mathcal{O}_{X}} \mathcal{F} \rightarrow \mathcal{O}_{X}(H+\mathbf{n} A) \otimes_{\mathcal{O}_{X}} \mathcal{F} \rightarrow \mathcal{O}_{H}(H+\mathbf{n} A) \otimes_{\mathcal{O}_{H}} \mathcal{F}_{\mid H} \rightarrow 0,
$$

from which we obtain

$$
h^{i}\left(X, \mathcal{O}_{X}(\mathbf{n} A) \otimes_{\mathcal{O}_{X}} \mathcal{F}\right) \leq h^{i-1}\left(H, \mathcal{O}_{H}(H+\mathbf{n} A) \otimes_{\mathcal{O}_{H}} \mathcal{F}_{\mid H}\right)=O\left(\max _{1 \leq j \leq \ell} n_{j}^{d-i}\right)
$$

for any integer $i \geq 1$ by induction. Since the Euler characteristic

$$
\chi\left(\mathcal{O}_{X}(\mathbf{n} A) \otimes_{\mathcal{O}_{X}} \mathcal{F}\right)=\sum_{i=0}^{d}(-1)^{i} h^{i}\left(X, \mathcal{O}_{X}(\mathbf{n} A) \otimes_{\mathcal{O}_{X}} \mathcal{F}\right)
$$

is a polynomial of total degree at most $d$ in $n_{1}, \ldots, n_{\ell}[10$, I.1], we deduce that $h^{0}\left(X, \mathcal{O}_{X}(\mathbf{n} A) \otimes_{\mathcal{O}_{X}} \mathcal{F}\right)=O\left(\max _{1 \leq j \leq \ell} n_{j}^{d}\right)$ and the lemma is proved.

\section{Proof of Theorem 1.1}

Let $X$ be a projective variety of dimension $d \geq 1$ and let $D \in \operatorname{Div}(X)_{\mathbb{R}}$ be an ample $\mathbb{R}$-Cartier $\mathbb{R}$-divisor. We fix a closed proper subscheme $Z \varsubsetneqq X$, and we denote by $\pi_{Z}: \widetilde{X} \rightarrow X$ the blow-up of $X$ along the ideal sheaf $I_{Z}$ defining $Z$ in $X$. We let $E$ be the exceptional divisor and for any $t \in \mathbb{R}$, we consider the $\mathbb{R}$-Cartier $\mathbb{R}$-divisor $D_{t}=\pi_{Z}^{*} D-t E$ on $\widetilde{X}$. Recall that the Seshadri constant of $D$ along $Z$ is

$$
\epsilon_{Z}(D)=\sup \left\{t \in \mathbb{R}_{\geq 0} \mid D_{t} \text { is ample }\right\}
$$


Since $\mathcal{O}_{\widetilde{X}}(-E)=\mathcal{O}_{\widetilde{X}}(1)$ is relatively ample with respect to $\pi_{Z}, \epsilon_{Z}(D)>0$ is positive by $\left[12\right.$, Proposition 1.7.10]. We also consider the invariant $\omega_{Z}(D) \in[0, \infty]$ defined by

$$
\omega_{Z}(D)=\sup \left\{t \in \mathbb{R}_{\geq 0} \mid D_{t \mid E} \text { is ample }\right\}
$$

where by a slight abuse of notation we denoted by $D_{t \mid E}$ the image in $N^{1}(E)_{\mathbb{R}}$ of the class of $D_{t}$ in $N^{1}(X)_{\mathbb{R}}$. Note that $\omega_{Z}(D) \geq \epsilon_{Z}(D)$. The goal of this section is to prove the following result, which generalizes Theorem 1.1 in the introduction.

Theorem 3.1. We have

$$
\epsilon_{Z}(D)=\sup \left\{t \in\left[0, \omega_{Z}(D)\right) \mid \varphi_{D, Z} \text { is polynomial on }[0, t]\right\} \text {. }
$$

Note that when $\mathcal{O}_{E}(-E)$ is nef, then $\omega_{Z}(D)=\infty$. Hence Theorem 3.1 indeed implies Theorem 1.1. We shall derive it from the following proposition.

Proposition 3.2. We have

$$
\begin{aligned}
\epsilon_{Z}(D) & =\sup \left\{t \in\left[0, \omega_{Z}(D)\right) \mid \operatorname{vol}\left(D_{t}\right)=D_{t}^{d}\right\} \\
& =\sup \left\{t \in\left[0, \omega_{Z}(D)\right) \mid \widehat{h}^{1}\left(\widetilde{X}, D_{t}\right)=0\right\}
\end{aligned}
$$

When $Z=\{x\}$ is a point, this statement is implicitly proved by McKinnon and Roth in [13, Proof of Theorem 9.1]. We shall prove Proposition 3.2 by combining the arguments of [13] with Lemma 2.2. We need the following lemma, which improves and generalizes [13, Lemma 4.1].

Lemma 3.3. For any integer $i \in[2, d]$ and any real number $t \in\left[0, \omega_{Z}(D)\right]$, we have $\widehat{h}^{i}\left(\widetilde{X}, D_{t}\right)=0$. Moreover,

$$
\operatorname{vol}\left(D_{t}\right)=D_{t}^{d}+\widehat{h}^{1}\left(\widetilde{X}, D_{t}\right) .
$$

Proof. We first assume that $D \in \operatorname{Div}(X)$ is a Cartier divisor. Let $i \in\{2, \ldots, d\}$ and $t \in\left[0, \omega_{Z}(D)\right]$. In order to prove that $\widehat{h}^{i}\left(\widetilde{X}, D_{t}\right)=0$, we may assume that $t \in\left(0, \omega_{Z}(D)\right) \cap \mathbb{Q}$ by continuity of $\widehat{h}^{i}$. By homogeneity of $\widehat{h}^{i}$, we may even assume that $t$ is a positive integer. For any integer $n \geq 1$, we have an exact sequence

$$
0 \rightarrow \mathcal{O}_{\widetilde{X}}\left(n D_{t}\right) \rightarrow \mathcal{O}_{\widetilde{X}}\left(n D_{0}\right) \rightarrow \mathcal{O}_{\widetilde{X}}\left(n D_{0}\right) \otimes_{\mathcal{O}_{\widetilde{X}}} \mathcal{O}_{n t E} \rightarrow 0,
$$

where $n t E$ denotes the subscheme of $\widetilde{X}$ defined by the $n t$-th power of the ideal sheaf $I_{E}$ of the Cartier divisor $E$. Since $D$ is ample, we have $H^{j}\left(\widetilde{X}, \mathcal{O}_{\widetilde{X}}\left(n D_{0}\right)\right)=0$ for all $n \gg 1$ and $j>0$. It follows from (2) that

$$
H^{i}\left(\tilde{X}, n D_{t}\right)=H^{i-1}\left(\widetilde{X}, \mathcal{O}_{\widetilde{X}}\left(n D_{0}\right) \otimes \mathcal{O}_{n t E}\right),
$$

for $n$ large enough. For any integer $\ell \geq 1$ we have $\mathcal{O}_{E}(-\ell E)=I_{E}^{\ell} / I_{E}^{\ell+1}$. This yields an exact sequence of sheaves on $\widetilde{X}$

$0 \rightarrow \mathcal{O}_{\widetilde{X}}\left(n D_{0}\right) \otimes_{\mathcal{O}_{\widetilde{X}}} \mathcal{O}_{E}(-\ell E) \rightarrow \mathcal{O}_{\widetilde{X}}\left(n D_{0}\right) \otimes_{\mathcal{O}_{\widetilde{X}}} \mathcal{O}_{(\ell+1) E} \rightarrow \mathcal{O}_{\widetilde{X}}\left(n D_{0}\right) \otimes_{\mathcal{O}_{\widetilde{X}}} \mathcal{O}_{\ell E} \rightarrow 0$ from which we obtain

$h^{i-1}\left(\widetilde{X}, \mathcal{O}_{\widetilde{X}}\left(n D_{0}\right) \otimes \mathcal{O}_{(\ell+1) E}\right) \leq h^{i-1}\left(\widetilde{X}, \mathcal{O}_{\widetilde{X}}\left(n D_{0}\right) \otimes \mathcal{O}_{\ell E}\right)+h^{i-1}\left(E, \mathcal{O}_{E}\left(n D_{0}-\ell E\right)\right)$.

Combining this inequality with $(3)$, we get

$$
h^{i}\left(\widetilde{X}, n D_{t}\right)=h^{i-1}\left(\widetilde{X}, \mathcal{O}_{\widetilde{X}}\left(n D_{0}\right) \otimes \mathcal{O}_{n t E}\right) \leq \sum_{\ell=0}^{n t-1} h^{i-1}\left(E, \mathcal{O}_{E}\left(n D_{0}-\ell E\right)\right) .
$$

Let $0 \leq \ell \leq n t-1$ be an integer and let $k=\lceil\ell / t\rceil$ be the least integer with $k \geq \ell / t$. We have

$$
\mathcal{O}_{E}\left(n D_{0}-\ell E\right)=\mathcal{O}_{E}\left((n-k) D_{0}+k D_{t}+j E\right),
$$


where $j=t k-\ell \in\{0, \cdots, t\}$. Since $\mathcal{O}_{E}\left(D_{0}\right)$ and $\mathcal{O}_{E}\left(D_{t}\right)$ are nef, Lemma 2.2 applied with $\mathcal{F}=\mathcal{O}_{E}(j E)$ for all possible values of $j$ gives a real number $C>0$ such that

$$
h^{i-1}\left(E, \mathcal{O}_{E}\left(n D_{0}-\ell E\right)\right) \leq C n^{d-i}
$$

for all $n \in \mathbb{N}$ and $\ell \in\{0, \ldots, n t-1\}$. By (4), it follows that

$$
\widehat{h}^{i}\left(\widetilde{X}, D_{t}\right)=\lim _{n \rightarrow+\infty} \frac{h^{i}\left(X, n D_{t}\right)}{n^{d} / d !}=0 .
$$

By the asymptotic Riemann-Roch theorem, we have $\operatorname{vol}\left(D_{t}\right)-\widehat{h}^{1}\left(\widetilde{X}, D_{t}\right)=D_{t}^{d}$, and the lemma is proved in the case where $D \in \operatorname{Div}(X)$.

In general, there exist Cartier divisors $D_{1}, \ldots, D_{\ell} \in \operatorname{Div}(X)$ such that $D \in V:=$ $\operatorname{Span}_{\mathbb{R}}\left(D_{1}, \ldots, D_{\ell}\right)$, and there exists a sequence $\left(D_{n}\right)_{n \in \mathbb{N}}$ in $\operatorname{Span}_{\mathbb{Q}}\left(D_{1}, \ldots, D_{\ell}\right) \subseteq V$ converging to $D$ in $V$. Let $t \in\left(0, \omega_{Z}(D)\right)$ be a real number. Then $D_{t \mid E}$ is ample, and therefore $D_{n, t_{\mid E}}$ is ample for $n$ sufficiently large by [12, Example 1.3.14]. In particular, $t \in\left(0, \omega_{Z}\left(D_{n}\right)\right]$ for $n \gg 1$. By the above and by homogeneity of the $\widehat{h}^{i}$, we have

$$
\operatorname{vol}\left(D_{n, t}\right)-\widehat{h}^{1}\left(\widetilde{X}, D_{n, t}\right)=D_{n, t}^{d} \text { and } \widehat{h}^{i}\left(D_{n, t}\right)=0 \forall i \in\{2, \ldots, d\}
$$

for $n \gg 1$. By continuity, (5) also holds for $D$ and the lemma is proved.

Proposition 3.2 is a consequence of Theorem 2.1 and Lemma 3.3.

Proof of Proposition 3.2. The second equality is implied by the first one by using Lemma 3.3. For any $t \in\left[0, \epsilon_{Z}(D)\right], D_{t}$ is nef and therefore $\operatorname{vol}\left(D_{t}\right)=D_{t}^{d}$ by $[12$, Corollary 1.4.41]. Since $t \leq \omega_{Z}(D)$, this gives the inequality

$$
\epsilon_{Z}(D) \leq \sup \left\{t \in\left[0, \omega_{Z}(D)\right] \mid \operatorname{vol}\left(D_{t}\right)=D_{t}^{d}\right\} .
$$

To prove the converse, let $\alpha$ be the supremum on the right hand side. By Lemma 3.3, we have $\widehat{h}^{1}\left(D_{t}\right)=0$ for all $t \in[0, \alpha)$. Let $s \in\left(0, \epsilon_{Z}(D)\right)$ and $t \in(0, \alpha)$ be real numbers. By definition of $\epsilon_{Z}(D), D_{s}$ is ample. For any $\lambda>0$ small enough we have $0 \leq \frac{t-\lambda s}{1-\lambda}<\alpha$, and by homogeneity of $\widehat{h}^{1}$ we deduce that

$$
\widehat{h}^{1}\left(\widetilde{X}, D_{t}-\lambda D_{s}\right)=(1-\lambda)^{d} \widehat{h}^{1}\left(\widetilde{X}, D_{\frac{t-\lambda s}{1-\lambda}}\right)=0 .
$$

By homogeneity and Lemma 3.3, we also have $\widehat{h}^{i}\left(\widetilde{X}, D_{t}-\lambda D_{s}\right)=0$ for all $i \geq 2$. By Theorem 2.1 $D_{t}$ is ample, hence $t \leq \epsilon_{Z}(D)$. By letting $t$ tend to $\alpha$, we obtain

$$
\sup \left\{t \in\left[0, \omega_{Z}(D)\right] \mid \operatorname{vol}\left(D_{t}\right)=D_{t}^{d}\right\}=\alpha \leq \epsilon_{Z}(D) .
$$

We can easily derive Theorem 3.1 from Proposition 3.2 as follows. Recall that $\varphi_{D, Z}(t)=\operatorname{vol}\left(D_{t}\right)$ for all $t \in \mathbb{R}_{\geq 0}$.

Proof of Theorem 3.1. By Proposition 3.2, it suffices to show that the following implication holds for any $\gamma>0$ :

$$
\varphi_{D, Z} \text { is polynomial on }[0, \gamma] \Longrightarrow \forall t \in[0, \gamma], \varphi_{D, Z}(t)=D_{t}^{d} .
$$

Let $\gamma>0$ be a real number such that $\varphi_{D, Z}[0, \gamma]$ is a polynomial. Then

$$
P: t \mapsto \varphi_{D, Z}(t)-D_{t}^{d}
$$

is a polynomial function on $[0, \gamma]$. On the other hand, $P(t)=0$ for all $t \in\left[0, \epsilon_{Z}(D)\right]$. Since $\epsilon_{Z}(D)>0$, it follows that $P=0$ on $[0, \gamma]$.

In the end of this section, we study in more detail the case where $Z \varsubsetneqq X$ is a smooth irreducible curve. The following example gives an alternative description of the invariant $\omega_{Z}(D)$. 
Example 3.4. (1) Assume that $X$ is a smooth surface and that $Z=C \varsubsetneqq X$ is a smooth irreducible curve. In that case, $\widetilde{X}=X$ and $E=C$. In particular, $\mathcal{O}_{E}(-E)$ is nef if and only if $C^{2} \leq 0$. On the other hand, $D_{t \mid E}$ is ample if and only if $D_{t} \cdot E=D \cdot C-t C^{2}>0$. It follows that $\omega_{C}(D)=\infty$ if $C^{2} \leq 0$, and $\omega_{C}(D)=(D \cdot C) / C^{2}$ otherwise.

(2) Assume that $X$ is smooth and that $Z=C \varsubsetneqq X$ is a smooth irreducible curve. In that case, the normal sheaf $\mathcal{N}_{C}:=\left(I_{C} / I_{C}^{2}\right)^{\vee}$ is a vector bundle of rank $d-1$ on $C$ and we have $E=\operatorname{Proj}\left(\mathcal{N}_{C}^{\vee}\right)$. We denote by $\mu_{\max }\left(\mathcal{N}_{C}\right)$ the maximal slope of $\mathcal{N}_{C}$, defined as

$$
\mu_{\max }\left(\mathcal{N}_{C}\right)=\max _{0 \neq \mathcal{F} \subseteq \mathcal{N}_{C}} \frac{\operatorname{deg}(\mathcal{F})}{\operatorname{rk}(\mathcal{F})},
$$

where $\mathcal{F}$ runs over the non-zero sub-bundles of $\mathcal{N}_{C}$. We also define the strong maximal slope of $\mathcal{N}_{C}$ as $\bar{\mu}_{\max }\left(\mathcal{N}_{C}\right)=\sup _{\tau: C^{\prime} \rightarrow C} \mu_{\max }\left(\tau^{*} \mathcal{N}_{C}\right)$, where the supremum is over the finite $k$-morphisms $\tau: C^{\prime} \rightarrow C$ of smooth projective integral curves (when $k$ has characteristic zero, we have $\bar{\mu}_{\text {max }}\left(\mathcal{N}_{C}\right)=$ $\mu_{\max }\left(\mathcal{N}_{C}\right)$ by $[14$, Proposition $\left.7.1(3)]\right)$. Let $\xi \in N^{1}(E)_{\mathbb{R}}$ be the class of $\mathcal{O}_{E}(1)$ and let $f \in N^{1}(E)_{\mathbb{R}}$ be the class of a fiber of $E \rightarrow C$. For any $t \in \mathbb{R}_{\geq 0}$, the class of $D_{t \mid E}$ in $N^{1}(E)_{\mathbb{R}}$ is $(D \cdot C) f+t \xi$ for any $t \geq 0$. By $[14$, Proposition $7.1(3)], D_{t \mid E}$ is ample if and only if $(D \cdot C)>t \bar{\mu}_{\max }\left(\mathcal{N}_{C}\right)$, and therefore

$$
\omega_{C}(D)= \begin{cases}(D \cdot C) / \bar{\mu}_{\text {max }}\left(\mathcal{N}_{C}\right) & \text { if } \bar{\mu}_{\text {max }}\left(\mathcal{N}_{C}\right)>0, \\ \infty & \text { if } \bar{\mu}_{\text {max }}\left(\mathcal{N}_{C}\right) \leq 0 .\end{cases}
$$

We refer the reader to [16, section 3] for more explicit computations of the invariant $\omega_{C}(D)$ in the case where $X=\mathbb{P}_{k}^{3}$.

In view of Example 3.4 (1), we see that the assumption of Theorem 1.1 is rather restrictive when $\operatorname{dim} Z>0$. Nevertheless, the following corollary shows that when $Z=C$ is a smooth irreducible curve in a smooth surface, Theorem 1.1 and Corollary 1.3 remain valid even if $\mathcal{O}_{E}(-E)$ is not nef. Recall that $\phi_{D, C}(t)=-\varphi_{D, C}^{\prime}(t) / d$ for every $t \geq 0$ such that $\varphi_{D, C}(t)>0$.

Corollary 3.5. Assume that $X$ is a smooth surface and that $Z=C \varsubsetneqq X$ is a smooth irreducible curve. Then

$$
\begin{aligned}
\epsilon_{C}(D) & =\sup \left\{t \geq 0 \mid \varphi_{D, C} \text { is polynomial on }[0, t]\right\} \\
& =\sup \left\{t \geq 0 \mid \phi_{D, C} \text { is differentiable on }(0, t)\right\} .
\end{aligned}
$$

Proof. If $C^{2} \leq 0$, then $\mathcal{O}_{E}(-E)$ is nef and the result is given by Theorem 1.1 and Corollary 1.3. In the following, we assume that $C^{2}>0$. In particular, $\omega_{C}(D)=$ $(D \cdot C) / C^{2}$ by Example $3.4(1)$. Let

$$
\beta_{C}(D):=\sup \left\{t \geq 0 \mid \varphi_{D, C} \text { is polynomial on }[0, t]\right\},
$$

and let $\gamma \in\left(0, \beta_{C}(D)\right)$ be a real number. Then $\varphi_{D, C}$ is a decreasing polynomial function on $[0, \gamma]$. As in the proof of Theorem 3.1, we have

$$
\varphi_{D, C}(t)=D_{t}^{2}=D^{2}-2 t D \cdot C+t^{2} C^{2}
$$

for every $t \in[0, \gamma]$. Since $\varphi_{D, C}(\gamma)=\operatorname{vol}\left(D_{\gamma}\right) \geq 0$, studying the variation of the polynomial $\varphi_{D, C}[[0, \gamma]$ leads to the inequalities

$$
\gamma \leq \frac{D \cdot C-\sqrt{(D \cdot C)^{2}-D^{2} C^{2}}}{C^{2}} \leq \frac{D \cdot C}{C^{2}}=\omega_{C}(D)
$$

(note that $(D \cdot C)^{2}-D^{2} C^{2} \geq 0$ by the Hodge index Theorem). Letting $\gamma$ tend to $\beta_{C}(D)$, we obtain $\beta_{C}(D) \leq \omega_{C}(D)$. On the other hand we have $\epsilon_{C}(D)=$ 
$\min \left\{\beta_{C}(D), \omega_{C}(D)\right\}$ by Theorem 3.1 , and therefore

$$
\epsilon_{C}(D)=\beta_{C}(D)=\sup \left\{t \geq 0 \mid \varphi_{D, C} \text { is polynomial on }[0, t]\right\} .
$$

Combining this equality with $[1$, Theorem $]$ as in the introduction, we also have

$$
\epsilon_{C}(D)=\sup \left\{t \geq 0 \mid \phi_{D, C} \text { is differentiable on }(0, t)\right\} \text {. }
$$

Remark 3.6. Assume that $X$ is smooth (of arbitrary dimension $d \geq 1$ ) and that $Z \varsubsetneqq X$ is a smooth divisor with Picard $\operatorname{rank} \rho(Z)=1$. In that case, we also have

$$
\epsilon_{Z}(D)=\beta_{Z}(D):=\sup \left\{t \geq 0 \mid \varphi_{D, Z} \text { is polynomial on }[0, t]\right\} .
$$

Indeed, if $t>\omega_{Z}(D)$ then $D_{t \mid Z}$ is not ample, hence it is not big since $\rho(Z)=1$. Arguing as in the proof of Lemma 3.3, it follows that $\varphi_{D, Z}(t)=\varphi_{D, Z}\left(t^{\prime}\right)$ for all $t^{\prime}>t$. This implies that $\omega_{Z}(D) \geq \beta_{Z}(D)$, and therefore $\epsilon_{Z}(D)=\beta_{Z}(D)$ by Theorem 3.1.

\section{VARIATION OF VOLUME FUNCTIONS IN FAMILIES}

We retain the notation of section 3 , and we assume that the field $k$ is uncountable. Our goal in this section is to study the behaviour of $\varphi_{D, Z}$ when the subscheme $Z \varsubsetneqq X$ varies in a flat family. The following proposition implies in particular that the functions $\varphi_{D, Z}$ all coincide for sufficiently general zero-dimensional subschemes of a given cardinality, as claimed in the introduction (see Proposition 4.2 below).

Proposition 4.1. Let $S$ be a Noetherian scheme locally of finite type over $k$, and let $\mathcal{Z} \varsubsetneqq X \times{ }_{k} S$ be a closed subscheme, flat over $S$. For any $s \in S(k)$, let $\mathcal{Z}_{s}$ be the fiber of $\mathcal{Z}$ above $s$. Then there exists a countable union $V=\cup_{n \in \mathbb{N}} V_{n} \varsubsetneqq S$ of proper subvarieties of $S$ such that

$$
\varphi_{D, \mathcal{Z}_{s}}(t)=\varphi_{D, \mathcal{Z}_{s^{\prime}}}(t)
$$

for all $t \in \mathbb{R}_{\geq 0}$ and $s, s^{\prime} \in S(k) \backslash V$.

Proof. We assume that $S$ is irreducible without loss of generality. We first consider the case where $D \in \operatorname{Div}(X)$ is a Cartier divisor. Let $I_{\mathcal{Z}} \subset \mathcal{O}_{X \times_{k} S}$ be the ideal sheaf defining $\mathcal{Z}$ in $X \times_{k} S$, and let $s \in S(k)$. We identify the fiber $X \times_{k}\{s\}$ with $X$ and we denote by $j_{s}: X \hookrightarrow X \times_{k} S$ the corresponding closed immersion. Let $\pi_{s}: \widetilde{X}_{s} \rightarrow X$ be the blow-up of $X$ along the ideal $I_{\mathcal{Z}_{s}}$ defining $\mathcal{Z}_{s}$ in $X$, and let $E_{s}$ be the exceptional divisor. By flatness, $j_{s}^{*} I_{\mathcal{Z}}=I_{\mathcal{Z}_{s}}$ is the ideal defining $\mathcal{Z}_{s}$ in $X$. By the semi-continuity theorem applied to $X \times_{k} S \rightarrow S$, the function

$$
s \in S(k) \mapsto h^{0}\left(X, \mathcal{O}_{X}(p D) \otimes_{\mathcal{O}_{X}} I_{\mathcal{Z}_{s}}^{q}\right)
$$

is upper semicontinuous for any integers $p, q \geq 0$. On the other hand, we have $\pi_{s *} \mathcal{O}_{\widetilde{X}_{s}}(-q E)=I_{\mathcal{Z}_{s}}^{q}$ for any sufficiently large integer $q$, and therefore the projection formula gives

$$
h^{0}\left(\widetilde{X}_{s}, p \pi_{s}^{*} D-q E_{s}\right)=h^{0}\left(X, \mathcal{O}_{X}(p D) \otimes I_{\mathcal{Z}_{s}}^{q}\right)
$$

for any integers $p, q \geq 0$ with $q$ large enough. It follows that

$$
\left\{s \in S(k) \mid \varphi_{D, \mathcal{Z}_{s}}(t)<\alpha\right\}
$$

is a countable intersection of open subsets in $S(k)$ for any $t \in \mathbb{Q} \geq 0$ and $\alpha \in \mathbb{R}$. This implies the existence of a countable union of proper subvarieties $V=\cup_{n \in \mathbb{N}} V_{n} \varsubsetneqq S$ such that for all $s \in S(k) \backslash V$, we have

$$
\forall t \in \mathbb{Q} \geq 0, \quad \varphi_{D, \mathcal{Z}_{s}}(t)=\operatorname{vol}\left(\pi_{s}^{*} D-t E_{s}\right)=\inf _{s^{\prime} \in S(k)} \varphi_{D, \mathcal{Z}_{s^{\prime}}}(t) .
$$


By continuity of vol, (6) actually holds for all $t \in \mathbb{R}_{>0}$. Therefore the function $\varphi_{D, \mathcal{Z}_{s}}$ is independent of the choice of $s \in S(k) \backslash V$, and the proposition is proved in the case where $D \in \operatorname{Div}(X)$ is a Cartier divisor.

In general, there exists a sequence $\left(D_{n}\right)_{n \in \mathbb{N}}$ of elements of $\operatorname{Div}(X) \otimes_{\mathbb{Z}} \mathbb{Q}$ such that $\left(D_{n}\right)_{n \in \mathbb{N}}$ converges to $D$ in $N^{1}(X)_{\mathbb{R}}$. By the above and by homogeneity of vol, for every $n \in \mathbb{N}$ there exists a countable union of proper subvarieties $W_{n} \varsubsetneqq S$ such that $\varphi_{D_{n}, \mathcal{Z}_{s}}(t)=\varphi_{D_{n}, \mathcal{Z}_{s^{\prime}}}(t)$ for all $t \in \mathbb{R}_{\geq 0}$ and all $s, s^{\prime} \in S(k) \backslash W_{n}$. Let $V=\cup_{n \in \mathbb{N}} W_{n}$ and let $s, s^{\prime} \in S(k) \backslash V$. Then for every $t \in \mathbb{R}_{\geq 0}$, we have

$$
\varphi_{D, \mathcal{Z}_{s}}(t)=\lim _{n \rightarrow \infty} \varphi_{D_{n}, \mathcal{Z}_{s}}(t)=\lim _{n \rightarrow \infty} \varphi_{D_{n}, \mathcal{Z}_{s^{\prime}}}(t)=\varphi_{D, \mathcal{Z}_{s^{\prime}}}(t)
$$

by continuity of vol.

Proposition 4.1 applies in particular when $S$ is a Hilbert scheme as follows. Given a very ample line bundle $\mathcal{O}_{X}(1)$ on $X$ and a polynomial $\Psi \in \mathbb{Q}[T]$, we denote by Hilb $_{X / k}^{\Psi}$ the Hilbert scheme of closed subschemes of $X$ whose Hilbert polynomial computed with respect to $\mathcal{O}_{X}(1)$ is $\Psi$. By Proposition 4.1 applied to $S=\operatorname{Hilb}_{X / k}^{\Psi}$, there exist a countable union of proper subvarieties $V_{\Psi} \varsubsetneqq \mathrm{Hilb}_{X / k}^{\Psi}$ and a function $\varphi_{D, \Psi}: \mathbb{R}_{\geq 0} \rightarrow \mathbb{R}$ such that

$$
\varphi_{D, Z}=\varphi_{D, \Psi}
$$

for every closed subscheme $Z \in \operatorname{Hilb}_{X / k}^{\Psi}(k) \backslash V_{\Psi}$. When we restrict our attention to zero-dimensional subschemes, we obtain the following result. Let $r \geq 1$ be an integer and let $X^{r}=X \times_{k} \cdots \times_{k} X$ be the fiber product of $r$ copies of $X$.

Proposition 4.2. There exist a countable union of proper subvarieties $V \varsubsetneqq X^{r}$ and a function $\varphi_{D, r}: \mathbb{R}_{\geq 0} \rightarrow \mathbb{R}$ such that $\varphi_{D,\left\{x_{1}, \ldots, x_{r}\right\}}=\varphi_{D, r}$ for all $\left(x_{1}, \ldots, x_{r}\right) \in$ $X^{r}(k) \backslash V$.

Proof. This follows from the above discussion by taking $\Psi=r$. Alternatively, this result follows directly from Proposition 4.1 applied to the schemes $S$ and $\mathcal{Z} \subset X \times{ }_{k} S$ defined by

$$
S(k)=\left\{\left(x_{1}, \cdots, x_{r}\right) \in X_{\mathrm{sm}}^{r}(k) \mid x_{i} \neq x_{j} \forall i \neq j\right\}
$$

and

$$
\mathcal{Z}(k)=\left\{\left(x,\left(x_{1}, \ldots, x_{r}\right)\right) \in X(k) \times S(k) \mid x \in\left\{x_{1}, \ldots, x_{r}\right\}\right\},
$$

where $X_{\mathrm{sm}}$ denotes the smooth locus of $X$.

Remark 4.3. As mentioned in the introduction, Theorem 1.1 and Proposition 4.2 imply that the function

$$
\left(x_{1}, \ldots, x_{r}\right) \in X^{r}(k) \mapsto \epsilon_{\left\{x_{1}, \ldots, x_{r}\right\}}(D) \in \mathbb{R}
$$

is constant outside a countable union of proper subvarieties $V \varsubsetneqq X^{r}$. This result is well-known and can also be derived directly from the definition of $\epsilon_{\left\{x_{1}, \ldots, x_{r}\right\}}(D)$, by using that ampleness is an open condition in a proper family of line bundles (see [12, Theorem 1.2.17 and Example 5.1.11]).

\section{ACKNOWLedgements}

I would like to thank the anonymous referees for their valuable comments and suggestions. 


\section{REFERENCES}

[1] Th. Bauer, A. Küronya, and T. Szemberg. Zariski chambers, volumes, and stable base loci. J. Reine Angew. Math., 576:209-233, 2004.

[2] Thomas Bauer, Sandra Di Rocco, Brian Harbourne, Michał Kapustka, Andreas Knutsen, Wioletta Syzdek, and Tomasz Szemberg. A primer on Seshadri constants. In Interactions of classical and numerical algebraic geometry, volume 496 of Contemp. Math., pages 33-70. Amer. Math. Soc., Providence, RI, 2009.

[3] Sébastien Boucksom, Charles Favre, and Mattias Jonsson. Differentiability of volumes of divisors and a problem of Teissier. J. Algebraic Geom., 18(2):279-308, 2009.

[4] José Ignacio Burgos Gil, Walter Gubler, Philipp Jell, Klaus Künnemann, and Florent Martin. Differentiability of non-archimedean volumes and non-archimedean Monge-Ampère equations. Algebr. Geom., 7(2):113-152, 2020. With an appendix by Robert Lazarsfeld.

[5] Steven Dale Cutkosky. Teissier's problem on inequalities of nef divisors. J. Algebra Appl., 14(9):1540002, 37, 2015

[6] Tommaso de Fernex, Alex Küronya, and Robert Lazarsfeld. Higher cohomology of divisors on a projective variety. Math. Ann., 337(2):443-455, 2007.

[7] Jean-Pierre Demailly. Singular Hermitian metrics on positive line bundles. In Complex algebraic varieties (Bayreuth, 1990), volume 1507 of Lecture Notes in Math., pages 87-104. Springer, Berlin, 1992.

[8] Thomas Eckl. Iterative dissection of Okounkov bodies of graded linear series on $\mathbb{C P}^{2}$, February 2015. Preprint, arXiv:1409.1736.

[9] L. Ein, R. Lazarsfeld, M. Mustaţă, M. Nakamaye, and M. Popa. Asymptotic invariants of line bundles. Pure Appl. Math. Q., 1(2, Special Issue: In memory of Armand Borel. Part 1):379-403, 2005.

[10] Steven L. Kleiman. Toward a numerical theory of ampleness. Annals of mathematics, 84(3):293-344, 1966.

[11] Alex Küronya. Asymptotic cohomological functions on projective varieties. Amer. J. Math., 128(6):1475-1519, 2006.

[12] Robert Lazarsfeld. Positivity in algebraic geometry. I, volume 48 of Ergebnisse der Mathematik und ihrer Grenzgebiete. 3. Folge. A Series of Modern Surveys in Mathematics [Results in Mathematics and Related Areas. 3rd Series. A Series of Modern Surveys in Mathematics]. Springer-Verlag, Berlin, 2004. Classical setting: line bundles and linear series.

[13] David McKinnon and Mike Roth. Seshadri constants, diophantine approximation, and Roth's theorem for arbitrary varieties. Invent. Math., 200(2):513-583, 2015.

[14] Atsushi Moriwaki. Relative Bogomolov's inequality and the cone of positive divisors on the moduli space of stable curves. J. Amer. Math. Soc., 11(3):569-600, 1998.

[15] Takumi Murayama. The gamma construction and asymptotic invariants of line bundles over arbitrary fields. Nagoya Math. J., 242:165-207, 2021.

[16] Roberto Paoletti. Seshadri constants, gonality of space curves, and restriction of stable bundles. J. Differential Geom., 40(3):475-504, 1994. 